\title{
Statistical Shape Modeling of Unfolded Retinotopic Maps for a Visual Areas Probabilistic Atlas
}

\author{
Isabelle Corouge $^{1}$, Michel Dojat ${ }^{2}$, and Christian Barillot ${ }^{1}$ \\ 1 Projet Vista, IRISA/INRIA-CNRS, Rennes, France \\ \{icorouge, cbarillo\}@irisa.fr \\ http://www.irisa.fr/vista \\ 2 INSERM, U594, CHU de Grenoble, Grenoble, France \\ Michel.Dojat@ujf-grenoble.fr \\ http://rhea.ujf-grenoble.fr/people/mdojat
}

\begin{abstract}
This paper proposes a statistical model of functional landmarks delimiting low level visual areas which are highly variable across individuals. Low level visual areas are first precisely delineated by fMRI retinotopic mapping which provides detailed information about the correspondence between the visual field and its cortical representation. The model is then built by learning the variability within a given training set. It relies on an appropriate data representation and on the definition of an intrinsic coordinate system to a visual map enabling to build a consistent training set on which a principal components analysis (PCA) is eventually applied. Our approach constitutes a first step toward a functional landmark-based probabilistic atlas of low level visual areas.
\end{abstract}

Keywords: Neuroimaging probabilistic atlas, visual areas, retinotopy, statistical modeling, PCA.

\section{Introduction}

In the context of neuroimaging probabilistic atlases, this paper focuses on the human visual cortex and more precisely on the statistical modeling of functional landmarks delimiting low level visual areas. The number of areas in the visual cortex, although estimated to be around 30, as well as their exact role in the distributed processing of visual information are not consensually determined yet. In this paper, we consider the following low order visual areas: V1, V2, V3, V3A and V4 of which a schematic 2D unfolded map can be found in Figure 1.a. As the denomination of these visual areas is still under debate, we adopt the notation presented in [1] where V4 is ventrally located along the ventral part of V3 in the inferior part of the occipital lobe. Note that "ventral" (resp. "dorsal") qualifies areas located below (resp. above) the calcarine sulcus. Besides, we will use suffixes "d" and "v" to designate the dorsal and ventral parts of V1, V2 and V3. These low order visual areas benefit from the retinotopic property: a univoque correspondence exists between the retina and its representation on the cortical surface of each of these areas. This forms an homeomorphism between a part of the visual field and the cortical surface within a given area. In other words, a two adjacent points stimulation in the visual field leads to a two neighboring points activation in the cortical surface. 


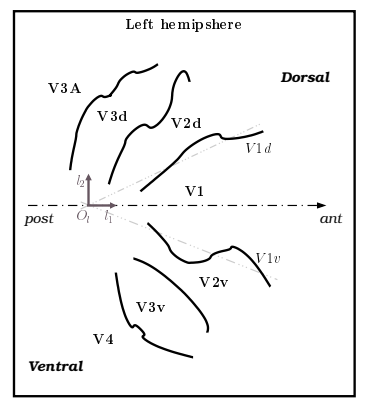

(a)

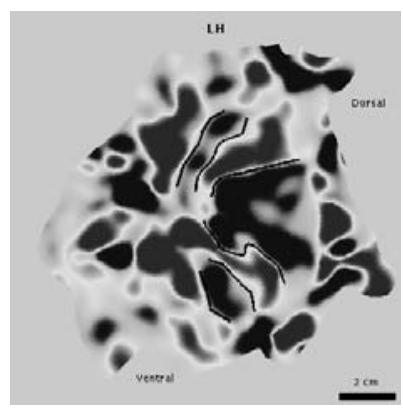

(b)

Fig. 1. (a) Schematic representation for the left hemisphere of an unfolded map of the considered visual areas. The coordinate system intrinsic to such a map is superimposed. (b) An unfolded 2D map showing VFR sign and visual areas borders (black lines) of one subject (left hemisphere).

Positions in the visual field are usually encoded by eccentricity and polar angle. A null eccentricity, which corresponds to the center of gaze, is represented at the occipital pole while representations of increasing eccentricities move toward more anterior parts of the cortical surface. The upper quadrant of the controlateral hemifield is projected below the calcarine sulcus (ventral areas) whereas the lower quadrant is projected above the calcarine sulcus (dorsal areas). V1, the primary visual area, receives information directly from the retina via the lateral geniculate nucleus and is located within the calcarine sulcus. The calcarine sulcus fundus is the cortical representation of the horizontal meridian. This latter also projects onto V2d/V3d and V2v/V3v borders. This alternates with the vertical meridian cortical representation located at V1/V2, V3d/V3A and V3v/V4 borders. Thus, the representation of the controlateral hemifield is mirrored between adjacent visual areas.

Retinotopic properties can be exploited to precisely delineate low order visual areas by fMRI retinotopic mapping [2], [3], [4]. Locations of these visual areas are highly variable across individuals [5]. In order to grasp this high inter-individual variability, we propose a statistical modeling of the functional landmarks delimiting low level visual areas, i.e. of the low level areas borders. We learn the variability within a given population and derive some statistics and shape occurrence probabilities from a statistical analysis, the principal component analysis (PCA). The training is based on a parametric representation of the structures of interest and on the definition of an intrinsic coordinate system for these structures in which instances of the training population can be matched.

In the following section, we describe the visual areas delineation process before building the statistical model in section 3. Experiments and results are presented in section 4 . Finally, in section 5 we discuss the proposed approach.

\section{Delineation of Low Level Visual Areas Borders}

The complete process to precisely delineate low level visual areas borders is described in details in [4]. We briefly recall the principle and the main steps of this process. It relies on retinotopic properties, periodic stimulation and exploits the orientation change of the representation of the local visual field between adjacent areas. It is performed by fMRI 
retinotopic mapping in three steps: measurements of functional data and 3D analysis, modeling of the cortical surface of interest and effective delineation.

Measurements of Functional Data and 3D Analysis. The visual stimuli used allow to determine the correspondence bewteen a position in the visual field and its representation onto the cortical surface. Four periodic stimuli are considered: a dilating/contracting ring and clockwise/counter-clockwise rotating wedges. In low level areas, these stimulations induce a periodic travelling wave in low level visual areas whose the phases are function of the position into the visual field. The corresponding periodic BOLD activation is recorded in 3D, a Fourier analysis is performed and then phase-maps are obtained.

Modeling of the Cortical Surface of Interest. The center of the grey matter, where functional activations are expected, is extracted from a tissue classification procedure followed by a dilatation of the grey matter/white matter interface. An unfolding algorithm [6] is applied to flatten the cortical surface, this one being restricted to the visual cortex area of interest. This algorithm enables to visualize the whole surface while resorting to no cuts.

Delineation of Visual Areas. A surface representation of the cortical response can now be obtained. Voxels are projected on the unfolded cortex map and projections are interpolated by a Gaussian smoothing. A measure called "local visual field ratio" (VFR) is eventually computed to perform delineation [7]:

$$
V F R=\frac{\partial\left(\phi_{r}, \phi_{\alpha}\right)}{\partial(u, v)}
$$

where $\left(\phi_{r}, \phi_{\alpha}\right)$ are the phases of observed responses corresponding to eccentricity $r$ and polar angle $\alpha$ of the visual field, the cortical surface being parameterized by $(u, v)$. The sign of VFR has a very precise interpretation: its change indicates a change in the orientation of the representation of the visual field. Therefore, visual areas borders correspond to zero level of VFR. Figure 1.b shows an example of such delineated maps. A good reproducibility has been demonstrated in [4].

\section{Statistical Modeling}

We propose now a statistical model of a delineated map, i.e. of the set of the six borders $\mathrm{V} 1 \mathrm{~d} / \mathrm{V} 2 \mathrm{~d}, \mathrm{~V} 1 \mathrm{v} / \mathrm{V} 2 \mathrm{v}, \mathrm{V} 2 \mathrm{~d} / \mathrm{V} 3 \mathrm{~d}, \mathrm{~V} 2 \mathrm{v} / \mathrm{V} 3 \mathrm{v}, \mathrm{V} 3 \mathrm{~d} / \mathrm{V} 3 \mathrm{~A}$ and V3v/V4. Our model is based on a training of the variability within a given population and is finally obtained by a wellknown multidimensional data analysis, the principal components analysis (PCA). The building of the training population first requires the definition of a data representation and second a matching scheme of these data. We assume relevant to establish a dense point to point correspondence between the set of maps and to derive theses correspondences from a parametric data representation once the population is aligned on a common coordinate system which has to be defined. In summary, building the training set consists 
in providing the data with a parametric representation, to sample this representation such that each of the six borders has the same number of sample points across the training set and to register this population on a common system of coordinates in which lines will be matched point to point.

\subsection{Data Parameterization and Resampling}

The delineation process produces visual borders areas as lines which are more or less straight or curved and irregularly sampled, even over-sampled. We have chosen to use cubic B-spline curves to fit them. This choice is indeed well adapted to model a large set of curves and enables an easy resampling of the data.

Let $n b p$ be the initial number of sample points of a given border $c$. A set of $n b c$ control points is first generated, from which it is then possible to generate a new set of $n b p^{r}$ points which regularly sample the border $c$. For each line, the number of sampled points, $n b p_{l, l=1, \ldots, 6}^{r}$, is chosen such that each border is provided with a satisfactory representation across all the subjects. If borders are initially represented with a similar initial number of sampled points, then each border is over-sampled on the most sampled instance. Otherwise, initially over-sampled borders are sub-sampled with $n b p^{r}$ performing a compromise of the number of sampled points of this border over the set of subjects. The choice of $n b p^{r}$ never leads to an effective sub-sampling of the initial border.

Each of the six borders of interest is thus represented by a set of $n b p_{l, l=1, \ldots, 6}^{r}$ points sampled on a cubic B-spline curve. Such a border can also be advantageously represented by the set of the $n b c_{l, l=1, \ldots, 6}^{r}$ control points associated with these new sampled points. This latter representation will be exploited in the statistical analysis step.

\subsection{Matching}

The alignment of the training set is based on the definition of an intrinsic coordinate system that we consider to be common to all instances in the training set. Let $\mathcal{R}_{l}\left(O_{l}, \mathbf{l}_{1}, \mathbf{l}_{2}\right)$ be this intrinsic coordinate system. $\mathcal{R}_{l}$ is determined for each instance in a geometric way only from V1 borders, i.e. from lines V1d/V2d and V1v/V2v. The confidence degree in the localization of $\mathrm{V} 1$ borders is indeed higher than in the other borders since the fMRI signal becomes less significant away from V1.

The $x$ axis is defined as the bissectrice of the axes of inertia of V1d/V2d and V1v/V2 $\mathrm{v}$ and follows the postero-anterior direction. Roughly, this axis separates ventral and dorsal areas and approximates the representation of horizontal meridian in V1. In other words, it follows the calcarine sulcus fundus. Axis $y$ is defined orthogonal to $x$ axis and is directed towards dorsal (resp. ventral) areas for the left (resp. right) hemisphere. The origin of $\mathcal{R}_{l}$ is defined as the intersection of the inertia axes of V1d/V2d and V1v/V2v, as illustrated in Figure 1.a.

The training population is then aligned on $\mathcal{R}_{l}$ by a change of basis from the intrinsic coordinate system to the original coordinate system of each subject $\mathcal{R}\left(O, \mathbf{i}_{1}, \mathbf{i}_{2}\right)$. This can be expressed by the rigid transformation (rotation + translation) matrix $\mathbf{M}$ :

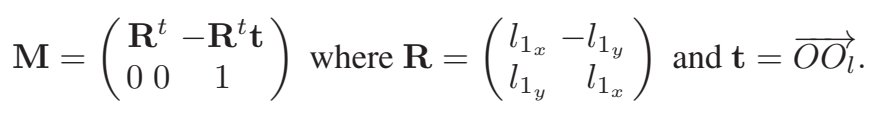



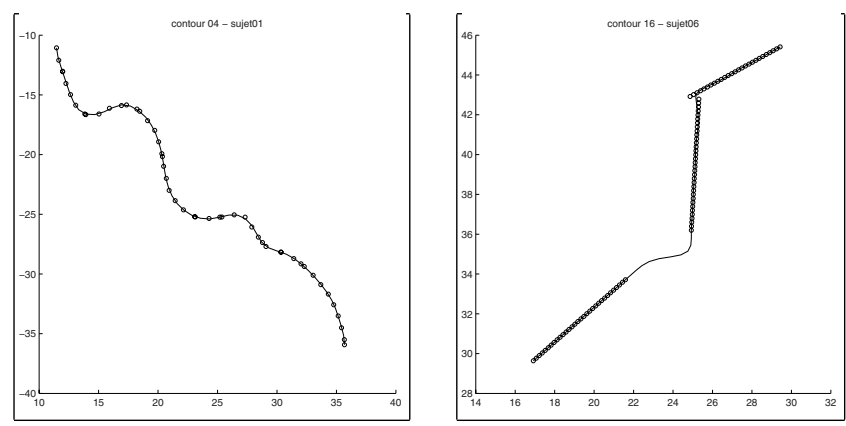

Fig. 2. Examples of resampled borders (continuous lines). Dots are the initial sampled points.

Once all the maps are expressed in $\mathcal{R}_{l}$, the matching consists in pairing control points having the same curvilinear abscissae.

\subsection{Statistical Analysis}

Let $\mathcal{P}$ be the training population made up of $N$ observations of maps $\mathbf{x}_{i, i=1, \ldots, N}$ resampled and registered as described above. Any map $\mathrm{x}$ is represented by the control points vector of the splines representing each of the six borders of interest:

$$
\mathbf{x}=\left(x_{0}^{1}, y_{0}^{1}, \ldots, x_{n b c_{1}^{r}-1}^{1}, y_{n b c_{1}^{r}-1}^{1}, \ldots, x_{0}^{6}, y_{0}^{6}, \ldots, x_{n b c_{6}^{r}-1}^{6}, y_{n b c_{6}^{r}-1}^{6}\right)^{t}
$$

where $\left(x_{i}^{l}, y_{i}^{l}\right)$ are the $n b c_{l}^{r}$ control points of the $l^{\text {th }}$ line of the map $(l=1, \ldots, 6)$.

We perform on $\mathcal{P}$ a principal components analysis. This analysis characterizes the variations within a given training population and extracts the principal modes of deformation relative to the mean map. Briefly, PCA consists in expressing the observations in a new basis, also orthogonal, with mean map $\overline{\mathbf{x}}$ as an origin and eigenvectors or modes of the observations covariance matrix $\mathbf{C}$ as axes. An approximation of these observations can be obtained by truncating a certain number of modes. The reconstructed observation is then written as $\mathbf{x}=\overline{\mathbf{x}}+\Phi_{\mathbf{m}} \mathbf{b}_{\mathbf{m}}$ where $m$ is the number of retained modes, $\Phi_{\mathbf{m}}$ the truncated modes matrix and $\mathbf{b}_{\mathbf{m}}=\left(b_{i}\right)_{i=1, \ldots, m}$ the $m$-dimensional vector representing the original observation in the truncated modal basis. Reconstruction quality can be measured by $\tau=\sum_{i=1}^{m} \lambda_{i} / \lambda_{T}$ where $\lambda_{i}$ is the $i^{\text {th }}$ eigenvalue, in decreasing order, of matrix $\mathbf{C}$ and corresponds to the variance explained by the $i^{\text {th }}$ mode, and where $\lambda_{T}$ is the total variance.

Under the assumption that the distribution of the elements of $\mathcal{P}$ is gaussian, the variation of $b_{i}, i=1, \ldots, m$ in an interval such as $\left[-3 \sqrt{\lambda_{i}}, 3 \sqrt{\lambda_{i}}\right]$ provides an indication of the variability of the studied object class. This indication is all the more reliable and realistic as the training set contains a sufficient number of representative instances.

\section{Experiments and Results}

Experiments have been led on an 8 subjects database. Since the extraction of the V3v/V4 right border has failed for one subject, only 7 subjects have been used for the right hemisphere. 
Table 1. For each border and each hemisphere, average and standard deviation in mm over all the subjects of the Hausdorff distance between initial lines ad resampled lines.

\begin{tabular}{|c||c|c|}
\hline Borders & Left hemisphere & Right hemisphere \\
\hline \hline V1d/V2d & $0.562 \pm 0.009$ & $0.561 \pm 0.004$ \\
\hline V1v/V2v & $0.603 \pm 0.010$ & $0.770 \pm 0.073$ \\
\hline V2d/V3d & $0.620 \pm 0.019$ & $0.571 \pm 0.005$ \\
\hline V2v/V3v & $0.612 \pm 0.026$ & $0.573 \pm 0.019$ \\
\hline V3d/V3A & $0.480 \pm 0.020$ & $0.586 \pm 0.011$ \\
\hline V3v/V4 & $0.522 \pm 0.016$ & $0.587 \pm 0.100$ \\
\hline
\end{tabular}

\subsection{Data Parameterization and Resampling}

Each border has been parameterized and resampled as described in section 3.1. The number $n b c$ of control points computed from the $n b p$ original sampled points has been fixed empirically such that $n b c=n b p / 2$. In theory, data representation is all the more fine as the ratio $n b c / n b p$ is close to 1 . In practise, due to the non-uniformity of the initial curve sampling, a ratio equal to 2 has appeared sufficient. Figure 2 shows some examples of resampled curves with initial curves superimposed. It indicates a very good conservation of the global original shape and of its position. In order to quantify the error induced by resampling, we have computed the Hausdorff distance between resampled lines and initial lines, for each border and each subject. Given 2 sets of points $S_{1}$ and $S_{2}$, the Hausdorff distance $H$ is defined as:

$$
H\left(S_{1}, S_{2}\right)=\max \left(h\left(S_{1}, S_{2}\right), h\left(S_{2}, S_{1}\right)\right) \text { with } h\left(S_{1}, S_{2}\right)=\max _{s_{1} \in S_{1}} \min _{s_{2} \in S_{2}}\left\|s_{1}-s_{2}\right\| .
$$

$h\left(S_{1}, S_{2}\right)$ is the directed Hausdorff distance from $S_{1}$ to $S_{2}, \|$. $\|$ being the Euclidean norm. The Hausdorff distance measures the distance from the point in $S_{1}$ the furthest to the point set $S_{2}$ and vice-versa. Hence, it is sensitive to a large error and provides somehow the maximal error resampling. The average of this distance over the set of subjects as well as its standard deviation are presented in Table 1. The error is weak and stable over the database.

\subsection{Alignment}

Figure 3 shows the borders locally registered onto the intrinsic coordinate system for both hemispheres. We can observe a relatively important variability within these populations, in particular as the position is concerned. This variability increases when moving away from $\mathrm{V} 1$.

\subsection{Statistical Analysis}

The statistical analysis has been applied on the control points of the splines defining the borders previously resampled and registered. The number of control points, $n b c_{l}^{r}$, has been fixed such that $n b c_{l}^{r} / n b p_{l}^{r}=4$. This choice induces an observation vector with 90 points and so 180 variables. It produces a fine representation of the lines to model while avoiding a huge dimensionality in regards to the cardinal of the training population. 

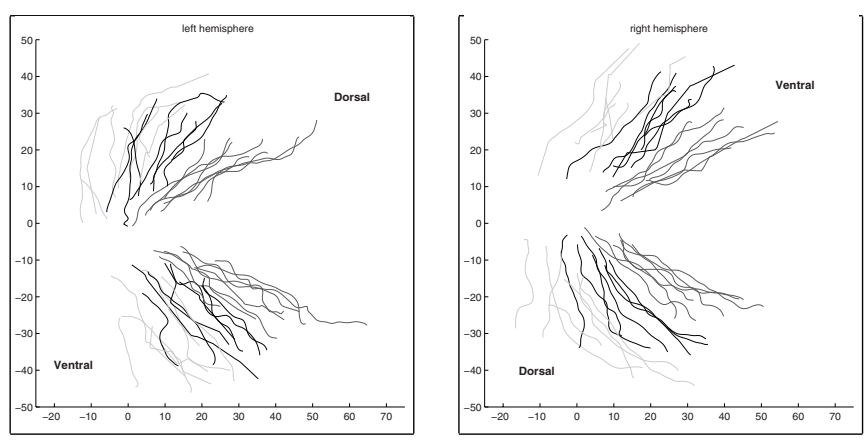

Fig. 3. Training populations corresponding to left and right hemispheres. They are locally registered in the intrinsic coordinate system. $\mathrm{V} 1 \mathrm{~d} / \mathrm{V} 2 \mathrm{~d}$ and $\mathrm{V} 1 \mathrm{v} / \mathrm{V} 2 \mathrm{v}$ are represented in dark grey, $\mathrm{V} 2 \mathrm{~d} / \mathrm{V} 3 \mathrm{~d}$ and $\mathrm{V} 2 \mathrm{v} / \mathrm{V} 3 \mathrm{v}$ in black, $\mathrm{V} 3 \mathrm{~d} / \mathrm{V} 3 \mathrm{~A}$ and $\mathrm{V} 3 \mathrm{v} / \mathrm{V} 4$ in light grey.

Table 2. Percentage, $\tau_{p}$, of cumulative variance according to the number of modes retained for left and right hemisphere; $\tau_{p}=\sum_{i=1}^{m} \lambda_{i} / \lambda_{T} \times 100$.

\begin{tabular}{|c||c|c|}
\hline Modes & Left hemisphere & Right hemisphere \\
\hline \hline 1 & 70.2 & 60.0 \\
\hline 2 & 84.1 & 82.6 \\
\hline 3 & 89.8 & 90.0 \\
\hline 4 & 94.2 & 95.1 \\
\hline 5 & 96.7 & 98.6 \\
\hline 6 & 98.8 & 100 \\
\hline 7 & 100 & 100 \\
\hline
\end{tabular}
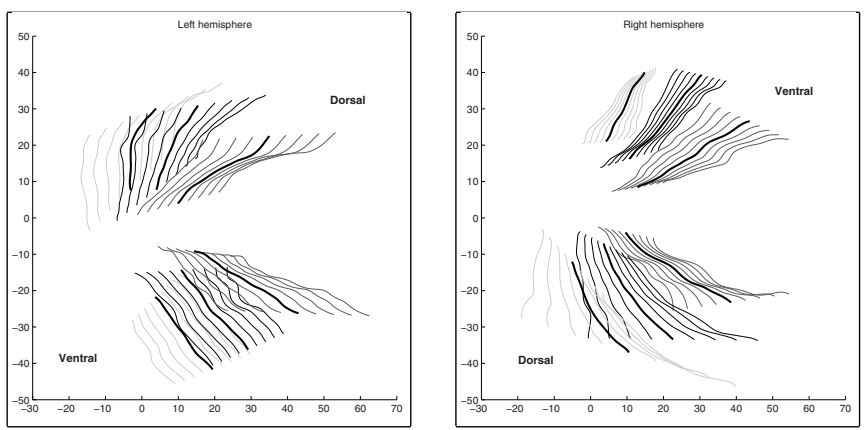

Fig. 4. For both hemispheres, variations according to the first mode around the mean map (thick black lines): $-2 \sqrt{\lambda_{1}} \leq b_{1} \leq+2 \sqrt{\lambda_{1}}$. V1d/V2d and V1v/V2v are represented in dark grey, $\mathrm{V} 2 \mathrm{~d} / \mathrm{V} 3 \mathrm{~d}$ and $\mathrm{V} 2 \mathrm{v} / \mathrm{V} 3 \mathrm{v}$ in black, V3d/V3A and V3v/V4 in light grey.

Table 2 exhibits the percentage of cumulative variance for each hemisphere according to the number of modes retained. The first mode strongly predominates. Figure 4 shows the variations around the mean map along the first mode. We observe a borders variability both in shape and position. However, position variability appears as the most important. We also note that borders appear less variable on the ventral side of the right hemisphere. 
These results, although interesting, should be interpreted in regards to the cardinal of the training population. In this restricted experimental framework, their scope is limited.

\section{Discussion and Perspectives}

We have proposed an approach to statistically model functional borders delimiting low order visual areas. Further work is still needed for a complete validation of such a model which namely requires to enlarge our data set. The definition of the intrinsic coordinate system has been constrained by presently available information. It could benefit from more anatomical and functional information. In particular, it would be interesting to effectively use the calcarine sulcus as a landmark. The origin, determined upon a geometric criterion, could also benefit from more functional information. For instance, it could be extracted from an area (measurements are marred with uncertainty and depend on SNR) responding to some predefined eccentricity and polarity. The use of the fovea representation on the cortical surface could also be investigated. Although this representation is not punctual, it could serve as a basis to define the origin. The matching process depends on the extraction technique, on the parameterization and on the definition of the intrinsic coordinate system. If the extraction partially failed such that some borders are more or less truncated, this matching is not adequate anymore and introduces a bias in the model. Two ways could be explored: improving the robustness of the extraction or using a based-distance matching, nevertheless relevance of this latter can not be systematically guaranteed. Eventually, the proposed method can be used in a given population 1) to study retinotopic area variations, 2) to realign functional scans based on functional visual landmarks or even on anatomical ones, e.g. calcarine sulcus, 3) to map inter-subject functional data onto the mean subject [8] and finally 4) to build a functional and anatomical probabilistic atlas of retinotopic areas for a given population [9]. Such an atlas could namely be exploited in mapping subjects for which only anatomical landmarks, e.g. calcarine sulcus, are available.

\section{References}

1. S. Zeki. Improbable areas in the visual brain. Trends in Neurosciences, 26(1): 23-26, 2003.

2. S.A. Engel, D.E. Rumelhart, B.A. Wandell, A.T. Lee, G.H. Glover, E.J. Chichilnisky, and M.N. Shadlen. fMRI of human visual cortex. Nature, 369(6481): 525, 1994.

3. M.I. Sereno, A.M. Dale, J.B. Reppas, K.K. Kwong, J.W. Belliveau, T.J. Brady, B.R. Rosen, and R.B.H. Tootell. Borders of multiple visual areas in human revealed by functional magnetic resonance imaging. Science, 268: 889-893, 1995.

4. J. Warnking, M. Dojat, A. Guérin-Dugué, C. Delon-Martin, S. Olympieff, N. Richard, A. Chéhikian, and C. Segebarth. fMRI retinotopic mapping - step by step. NeuroImage, 17(4): 1665-1685, 2002.

5. K. Amunts, A. Malikovic, H. Mohlberg, T. Schormann, and K. Zilles. Bordmann's areas 17 and 18 brought into stereotaxic space-Where and how variable? NeuroImage, 11: 66-84, 2000.

6. A. Guérin-Dugué, S. Olympieff, J. Gisert-Lopez, A. Chéhikian, J. Warnking, C. Rubin, and C. Segebarth. Représentation plane du cortex visuel en imagerie fonctionnelle à résonance magnétique. In RFIA, 2: 29-38, 2000.

7. M.I. Sereno, C.T. McDonald, and J.M. Allman. Analysis of retinotopic maps in extrastriate cortex. Cerebral Cortex, 4: 601-620, 1994. 
8. I. Corouge, P. Hellier, B. Gibaud, and C. Barillot. Inter-individual functional mapping: a non linear local approach. NeuroImage, 2003. In press.

9. D.C. Van Essen, J.W. Lewis, H.A. Drury, N. Hadjikhani, R.B.H. Tootell, M. Bakircioglu, and M.I. Miller. Mapping visual cortex in monkeys and humans using surface-based atlases. Vision Research, 41: 1359-1378, 2001. 